\title{
Extremal Bounds on Earthquake Movement from Geodetic Data: Application to the Landers Earthquake
}

\author{
by Hadley O. Johnson, Duncan Carr Agnew, and Ken Hudnut
}

\begin{abstract}
We present a technique to place quantifiable bounds on the moment of an earthquake from geodetic data, assuming known fault geometry. Application of this technique to the 1992 Landers earthquake shows that the moment must have been between 0.84 and $1.15 \times 10^{20} \mathrm{Nm}$ with $90 \%$ confidence ( $\mathrm{M}$ 7.25 to 7.34). We also find that to satisfy the data to this same level of confidence, the slip on the fault must have exceeded $7 \mathrm{~m}$ in at least one location, in good agreement with field mapping of the surface rupture.
\end{abstract}

\section{Introduction}

The release of stress in an earthquake causes both slip on the earthquake fault and deformation of the ground in the region around it; these are usually taken to be related through elastic dislocation theory. It is thus possible, in principle, to use the displacements of points at the ground surface to estimate characteristics of the subsurface slip on the fault. A variety of techniques have been employed for this (Ward and Barrientos, 1986; Harris and Segall, 1987; Segall and Harris, 1987; Du et al., 1992). We present a technique that is based on the same fundamental principles as these, but whose final product is a bound on the moment of the earthquake, rather than a model of the fault-slip distribution. Our technique is similar to the methods of Langbein (1981) and Vasco (1985). We have applied this technique to the 1992 Landers earthquake and present results from two different data sets, one from a preliminary set of measurements made soon after the earthquake, and the other from a much more extensive network of stations, including displacement results from many research organizations. This slightly unusual presentation is used to demonstrate in a more realistic way the ability of this technique to produce useful results both soon after and well after a large earthquake.

In their studies, Langbein (1981) and Vasco (1985) both used the linear programming (LP) framework to determine extremal fault-slip models, but each used the socalled infinity-norm as their measure of model misfit as opposed to the more typical two-norm (i.e., least squares). To minimize the infinity-norm misfit between predicted values and measured data means to minimize the maximum model misfit. That is, the modeled residual which is the worst fit to the actual measurements is minimized, a procedure that is extremely sensitive to data outliers. In this study we also use the LP framework, but use the one-norm as our measure of model misfit. The one-norm is more robust than either the two-norm or infinity-norm in the presence of noisy data, and is inherently much less variable as a statistical estimator than the infinity-norm (Parker, 1994).

\section{Technique}

To invert geodetic data for a subsurface slip distribution, we must first be able to perform the forward calculation relating fault slip to surface deformation. For this purpose, we model the earth as an elastic half-space. Standard methods exist for computing the response to slip on a dislocation surface for this model (Okada, 1985; Erickson, 1986). We have also assumed that the geometry of the fault is known; for the Landers earthquake this is a good assumption, given the coincidence of the surface rupture and the aftershock zone. Figure 1 shows the Landers surface rupture (K. Sieh, personal comm.); our simplified version of the fault trace is composed of 1-km-long straight segments and extends south of the Pinto Mountain fault to include the Eureka Peak fault, for a total length of $92 \mathrm{~km}$. The assumed fault plane extends from the surface to a depth of $15 \mathrm{~km}$, also in 1$\mathrm{km}$ increments, and dips $90^{\circ}$, resulting in a total of 1,380 model elements, each $1 \mathrm{~km}$ on a side. Figure 1 also shows the location and horizontal displacement vectors of the 11 geodetic stations from the preliminary data set, whose co-seismic displacements we seck to model (see Fig. 5 for a map view of the larger network).

Given the relative locations of the fault "patches" and geodetic stations, we begin the inversion process by calculating the displacements resulting from a unit-slip on each patch. (We have assumed that the slip was purely horizontal for the Landers event, though any slip direction, from thrust to normal, could have been considered.) The resulting Green's function matrix, $\mathbf{G}$, can then 


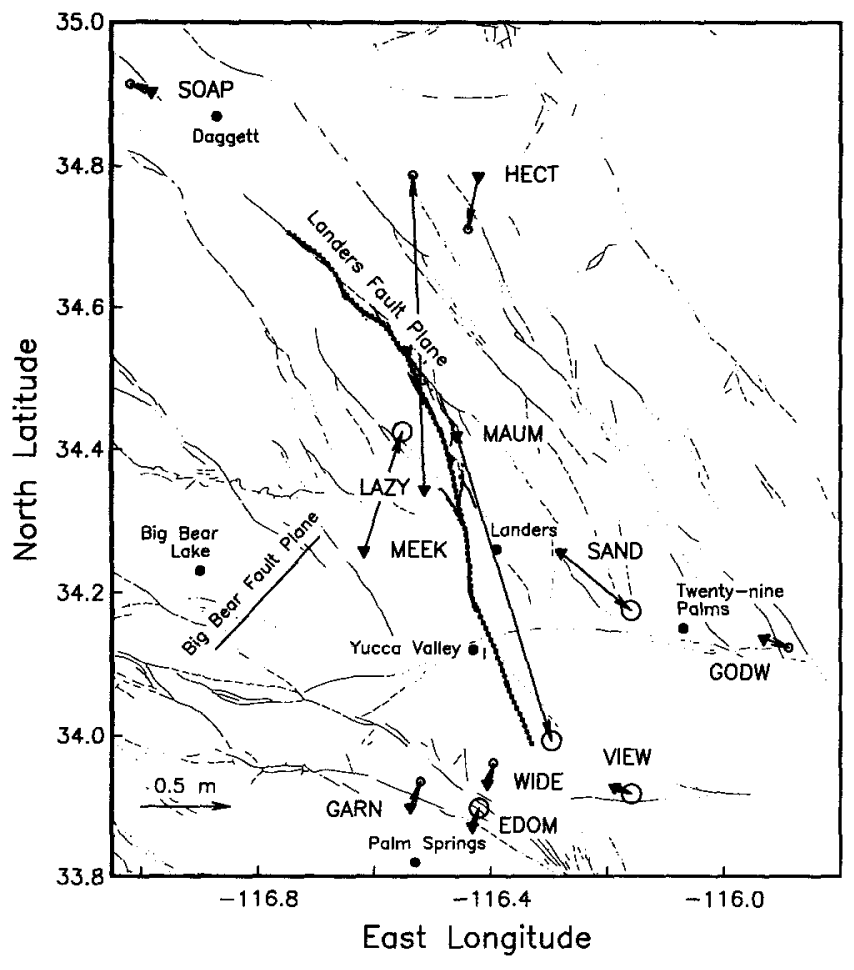

Figure 1. Overview of the Landers and Big Bear rupture area. The discretized fault plane used for this study is shown as 1-km-long segments extending for a total length of $92 \mathrm{~km}$. The Big Bear fault plane is defined by its aftershock distribution. The 11 geodetic stations which make up what we call the "small network" are shown along with their co-seismic displacements and $95 \%$ confidence regions.

be used to relate the slip model, $\hat{s}$, to the measured station displacements, $d$, by:

$$
d=\mathbf{G} \hat{s}+\epsilon,
$$

where $\epsilon$ is the discrepancy between the measured displacements and those predicted from $\hat{s}$.

Since the number of fault patches greatly exceeds the number of input data [there are a total of 27 data in the smaller network (Fig. 1) and 101 data in the larger network (Fig. 5)] there are an infinite number of slip models which can exactly reproduce the offsets; that is, satisfy equation (1) with $\epsilon=0$. In addition, since there are errors in the measured offsets, we should actually investigate all models that adequately match the data, not just those that match the data exactly-thus further expanding the range of acceptable models. There are a variety of ways to restrict the range of models in reasonable ways so that we determine a single, preferred, slip model (Matthews, 1991; Du et al., 1992; Parker, 1994). Here, instead of inverting for a single slip model, we wish to invert for a bound on a particular property of the slip model that all acceptable models must satisfy.
More specifically, we wish to find the largest and smallest possible moment consistent with the data. These bounds on moment will be valid for all possible slip models regardless of the kind of data used to construct these models (e.g., geodetic, seismic, strong motion, or combinations), recognizing that our technique is based on the following assumptions: that the earth may be modeled as a homogeneous elastic half-space, that the location of the fault plane is known, and that the fault slip was purely horizontal.

To determine these bounds, we add the following two sets of constraints to equation (1): that the elements of the slip model $\hat{s}$ are all positive (thus allowing only right-lateral slip) and that the moment be some set value $\boldsymbol{M}_{0}$. Our inversion problem is then stated:

$$
\begin{gathered}
\operatorname{minimize} F \equiv\left\|\mathbf{\Sigma}^{-1}(d-\mathbf{G} \hat{s})\right\|_{1} \\
\text { subject to } \mu A \sum_{i=1}^{n} \hat{s}_{i}=M_{0} \text { and } 0 \leqq \hat{s}_{i} \leqq \mathbf{u}_{i},
\end{gathered}
$$

where $\mathbf{\Sigma}$ is the covariance matrix (found from the estimated errors in the data), $A$ is the area of each fault patch $\left(10^{6} \mathrm{~m}^{2}\right), \mathbf{u}$ is a vector of upper bounds on the amount of slip allowed on each model element, and the model misfit is measured using the one-norm. Statistics for the one-norm misfit are not widely available, but a small table of values and a procedure for calculating others is contained in Parker and McNutt (1980). We next choose an appropriate confidence level, such as $90 \%$, and determine the misfit value corresponding to this probability. If the slip model that solves equations (2) and (3) produces a value of $F$ less than this acceptance level, then at least one slip model (and possibly an infinite number) exists that adequately predicts the measured data, is made up of patches of only right-lateral horizontal slip, and has the given moment. If the smallest model misfit is larger than this acceptance level, we can say no such model exists (at the $90 \%$ confidence level), and the assumed value of the moment is thus inconsistent with the data. Any of the constraints could, in fact, be called into question, but we believe the moment constraint is by far the least certain of the assumptions. By systematically varying the moment constraint, we can then determine the full range over which the problem has an acceptable solution at the chosen level of confidence.

Equations (2) and (3) are almost in the form of an LP problem, but a few modifications are required. In the canonical LP problem a linear functional of the model elements is minimized subject to a set of equality and inequality constraints. Note that there is no mention of a norm in this formulation. In particular, the one-norm in equation (2), with its absolute value function, is certainly not a linear functional of $\hat{s}$. By introducing two 
new sets of variables, however, we can bring the onenorm misfit in line with LP (Wagner, 1959). Let

$$
\begin{gathered}
\mathbf{\Sigma}^{-1}(d-\mathbf{G} \hat{s})=z^{\prime}-z^{\prime \prime} \\
0 \leqq z_{i}^{\prime} \\
0 \leqq z_{i}^{\prime \prime}
\end{gathered}
$$

so that the model residuals are written as the difference between two positive numbers. Now, instead of minimizing the norm in equation (2), we minimize the sum of $z^{\prime}$ and $z^{\prime \prime}$ and use equations (4) through (6) as additional constraints on the original problem [this counterintuitive procedure is discussed in Wagner (1959)]. The full problem is now written

$$
\begin{gathered}
\operatorname{minimize} F \equiv \sum_{i=1}^{m}\left(z_{i}^{\prime}+z_{i}^{\prime \prime}\right) \\
\text { subject to } \mathbf{\Sigma}^{-1} \mathbf{G} \hat{s}+z^{\prime}-z^{\prime \prime}=\mathbf{\Sigma}^{-1} d \\
\mu A \sum_{i=1}^{n} \hat{s}_{i}=M_{0} \\
0 \leqq \hat{s}_{i} \leqq \mathbf{u}_{i} \\
0 \leqq z_{i}^{\prime} \\
0 \leqq z_{i}^{\prime \prime},
\end{gathered}
$$

where the LP algorithm must now determine all $n$ elements of the slip model as well as the $m$ elements each of $z^{\prime}$ and $z^{\prime \prime}$-the one-norm is thus incorporated at the expense of adding $2 m$ new variables to the problem. As before, we can now sweep through a series of moment constraints looking for the points where $F$ in equation (7) is equal to the $90 \%$ confidence level for the one-norm statistic.

We have solved equations (7) through (10) using the LP algorithm contained in Numerical Recipes (Press $e t$ al., 1987, pp. 312-325), but this algorithm does not have the ability to incorporate upper bounds on the slip-model elements directly [right half of equation (10a)], and so these constraints must be explicitly added to the problem, resulting in a total of $n+m+1$ constraints instead of the $m+1$ represented by equations (8) and (9) [constraints in equations (10b) and (10c) are taken care of automatically by the LP code]. This slows the solution almost impossibly, and so in addition, we have used the BVLS code of Stark and Parker (1993) to solve this problem. The BVLS code is based on the NNLS algorithm of Lawson and Hanson (1974), but has been modified to incorporate model-constraint upper bounds automati- cally, thus speeding the solution greatly (by a factor of nearly 20 in this case). Unfortunately, BVLS is based on quadratic programming instead of linear programming. In a manner similar to our incorporation of the one-norm into the standard LP problem, it is possible to "fool" the BVLS algorithm into solving our linear problem by adding even more variables, as discussed in Stark and Parker (1993). This is our preferred method of solving equations (7) through (10).

As already stated, the studies of Langbein (1981) and Vasco (1985) used the infinity-norm as the measure of model misfit. For comparison with our one-norm results in the next section, we have implemented a version of LP incorporating the infinity-norm. In our notation, this problem is written as follows [compare with equations (1) through (4) of Langbein (1981)]:

$$
\begin{gathered}
\operatorname{minimize} F \equiv\left\|\mathbf{\Sigma}^{-1}(d-\mathbf{G} \hat{s})\right\|_{\infty} \\
\mu A \sum_{i=1}^{n} \hat{s}_{i}=M_{0} \\
0 \leqq \hat{s}_{i} .
\end{gathered}
$$

For the normalized infinity-norm misfit, the appropriate $90 \%$ confidence level for 27 data would be $F=2.887$. That is, all acceptable slip models will satisfy equations (12) and (13) and possess a "size," as measured by equation (11), of less than or equal to 2.887. (For a problem with $m$ data, and independent errors, we are interested in the joint probability that all $m$ residuals, as defined in equation (11), lie within their $90 \%$ confidence regions this is simply the product of the individual probabilities. In this case, the joint probability that $\mathbf{2 7}$ data lie within 2.887 standard deviations of their means is $90 \%$.)

\section{Results for a Small Geodetic Network}

The preliminary displacements of the 11 geodetic stations shown in Figure 1 were obtained from the U.S. Geological Survey (USGS) in Menlo Park and the Southern California Earthquake Center (SCEC) soon after the Landers earthquake in the summer of 1992. Five of these co-seismic offsets were measured by the USGS, using data from the global positioning system (GPS), and reduced using broadcast orbits for the satellites and the Bernese processing software. These offsets are relative to a station near Palm Springs, California (RESORTjust off the bottom edge of Fig. 1). The remaining six stations were monitored by SCEC and reduced using satellite orbits calculated from a global GPS network and the GAMIT processing software. These offsets are relative to the Permanent GPS Geodetic Array site at Piñon Flat Observatory (PFO) (Shimada and Bock, 1992). To bring these two sets of data into a common global ref- 
erence frame, we have used a model-predicted offset between the stations RESORT and PFO (PFO moved westnorthwest by about $0.5 \mathrm{~cm}$ relative to RESORT), and then used the results of Bock et al. (1993) for the co-seismic motion of station PFO relative to the global network. Because of the multiple ties and the preliminary nature of the GPS analyses, we have used conservative error estimates: $\pm 1 \mathrm{~cm}$ for both horizontal directions and \pm 3 $\mathrm{cm}$ vertically for the SCEC results, and $\pm 2.5 \mathrm{~cm}$ horizontally and $\pm 4 \mathrm{~cm}$ vertically for the USGS results.

The Big Bear earthquake (M 6.2), which occurred a few hours after the Landers earthquake, presents a complication to this procedure. Because all of the postearthquake GPS surveys were conducted after both earthquakes occurred, they actually measure the sum of the displacements from the earthquakes. We have experimented with several synthetic data sets to determine if the moments of the two earthquakes can be solved for simultaneously. This does not appear to be possible. Another option would be to remove the predicted deformation caused by the Big Bear event from the data before proceeding with the inversion. In light of the relative

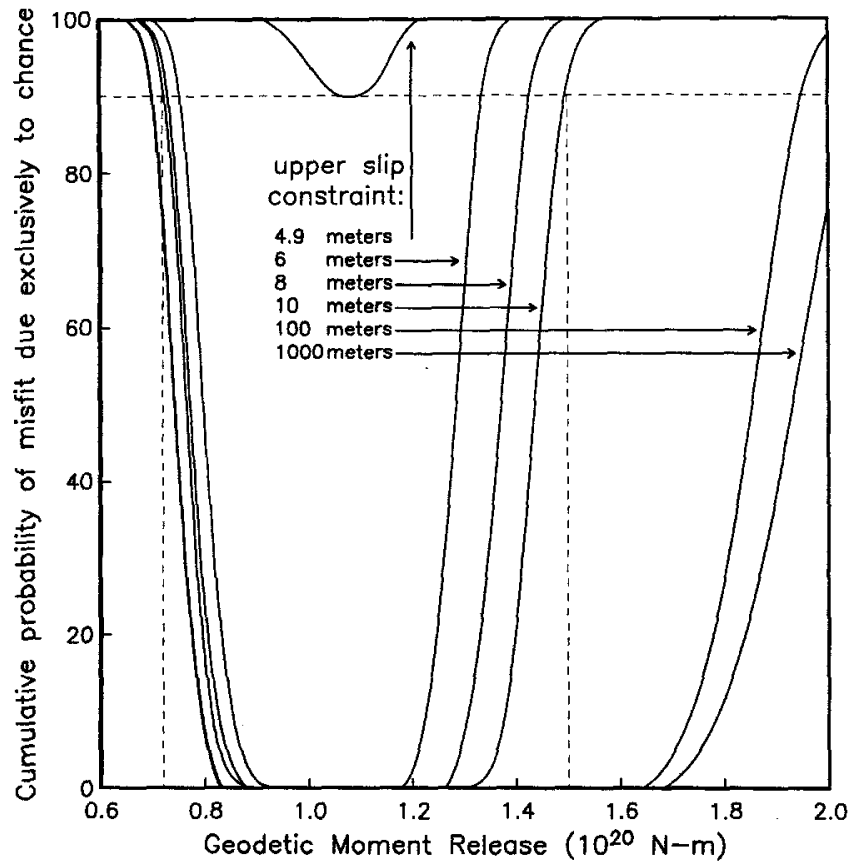

Figure 2. Slip-model probability plotted as a function of the total geodetic moment release for the small network. The vertical axis is the probability that the model misfit would be as small or smaller exclusively because of random fluctuations. Values plotted below the horizontal dashed line are thus statistically consistent with the uncertainties in the co-seismic offset data at the $90 \%$ level. The separate curves show the results for different upper limits imposed on the elements of the slip model. Taking $10 \mathrm{~m}$ of fault slip as a conservative upper limit, the resulting $90 \%$ confidence limit on moment is 0.72 to $1.5 \times 10^{20} \mathrm{Nm}$ (M 7.2 to 7.4 ). sizes of the two earthquakes (the Big Bear moment release was less than $4 \%$ of the Landers moment release) and the preliminary nature of the data, we have simply chosen to ignore the Big Bear event.

Figure 2 presents our primary result, in the form of slip-model confidence levels as a function of the earthquake moment $M_{0}$. The horizontal dashed line is at the $90 \%$ confidence level. Each curve gives the result for a different upper bound for the slip on all fault patches (equation 10a). As the upper bound is reduced from 1000 to $10 \mathrm{~m}$, and eventually to $4.9 \mathrm{~m}$, the resulting $90 \%$ confidence limits on the moment release (the range of moments over which the misfit curves fall below the $90 \%$ level) become smaller, until eventually the curve for a 4.9-m upper bound just dips to the $90 \%$ level. Depending on which upper limit we are willing to accept as the maximum slip that may have occurred (based on geological evidence from this and other similar events), we end up with different bounds on the moment. Taking the 10- $\mathrm{m}$ upper bound as a reasonable but still conservative level, the $90 \%$ bound on moment is about 0.72 to 1.5 $\times 10^{20} \mathrm{Nm}$ (M 7.2 to 7.4). The steepness of these curves shows that this result is quite insensitive to the confidence level used-the $95 \%$ limits would be nearly the same as the $50 \%$ limits.

Another conclusion we can draw from this figure,

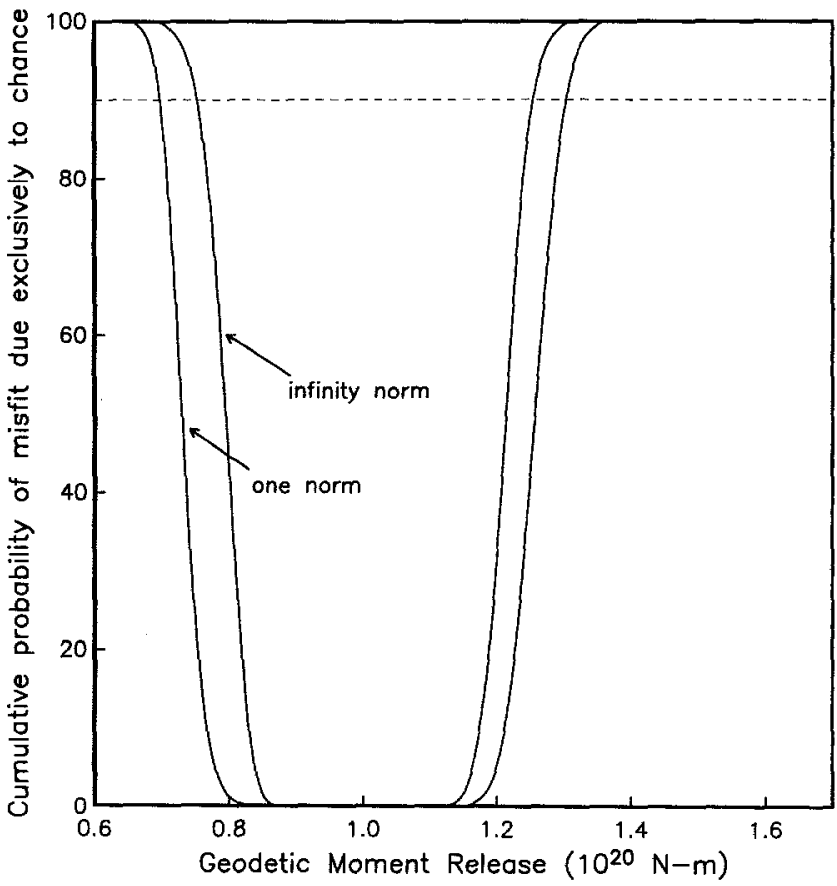

Figure 3. A comparison of the probability curves for the one-norm and infinity-norm for an upper-slip limit of $10 \mathrm{~m}$. The infinity-norm is inherently more variable as a statistical estimator and far less robust in the presence of data outliers. For these two reasons, the $90 \%$ confidence limits resulting from the one-norm calculations are preferred. 
also at the $90 \%$ confidence level, is that at least one fault patch must have slipped at least $4.9 \mathrm{~m}$ during the earthquake, although we cannot say exactly where this patch was located. This agrees well with the surface-slip data, which show a maximum offset of between 5 and $6 \mathrm{~m}$ at points along the rupture zone (Sieh et al., 1993), thus validating our computational methods to a degree.

For comparison, Figure 3 presents the difference between the moment limits for the one-norm and infinitynorm for a 10-m upper limit on each slip element. At the $90 \%$ level, the moment limits for the infinity-norm are 0.8 to $1.4 \times 10^{20} \mathrm{Nm}$; a smaller range than our onenorm result. These tight bounds are a result of the nature of the infinity-norm. Restricting the largest of the modeled residuals to be within some strict confidence interval severely restricts the "freedom" of the inversion routine when compared to the group-fit philosophy resulting from the one-norm technique. Why would we prefer the looser bounds of the one-norm over the tighter bounds of the infinity-norm? As previously mentioned, the answer lies in the robustness of the one-norm and its smaller inherent variability. The robustness argument is especially significant in this case, since we are working with preliminary data gathered by several different groups and only loosely tied together into a common reference frame.

To provide insight into how the inversion technique works, Figure 4 shows the best-fitting one-norm slip models at the lower and upper moment limit for a $10-\mathrm{m}$ upper bound on the maximum slip for all fault patches. While these slip models need bear no relationship whatsoever to the actual slip that occurred during the earthquake, they do help to understand the technique and also demonstrate the wide range of models which can fit the data. The distribution for the lower moment limit has all of the slip in two distinct clumps, with most of the slip near the center of the fault. This model is just able to adequately predict the measured surface displacements of the geodetic stations. For the upper moment limit we see a pattern of subsurface slip which is almost a mirror image, with the inversion trying to hide as much slip as possible at depth and at places along the length of the fault away from the geodetic data coverage, although some surface slip is still required near the center of the fault to satisfy the observations at stations MAUM and LAZY. As might be guessed, the upper moment limit is somewhat dependent on the maximum fault depth used in the

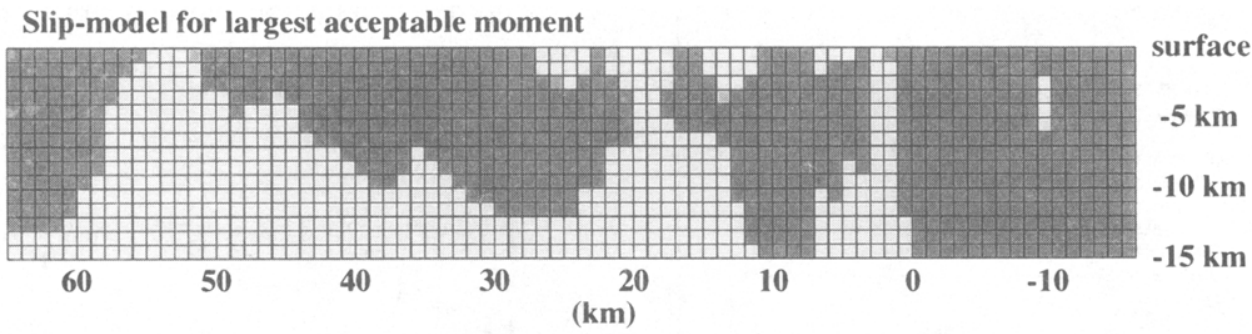

Slip-model for smallest acceptable moment

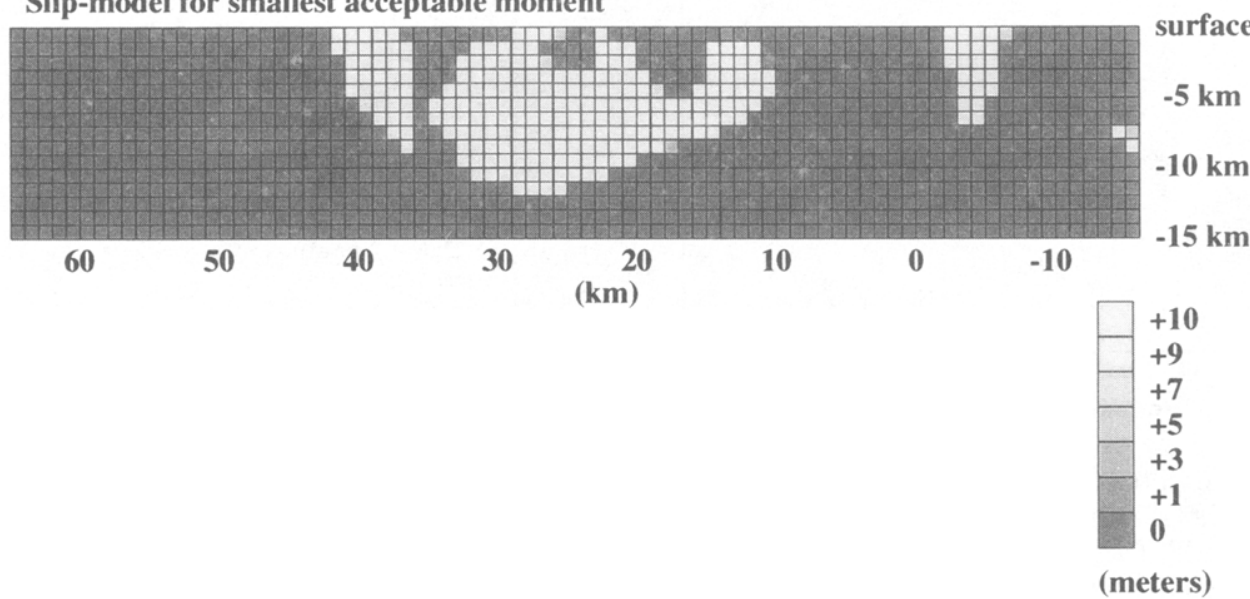

Figure 4. The slip models for the small network corresponding to the $90 \%$ confidence upper limit (top) and lower limit (bottom) on moment release, for the case where each individual element of the slip model is constrained to less than $10 \mathrm{~m}$ of slip. The view is from the southwest side of the fault plane, with zero located at the epicenter. In the lower panel, in order to match the data, the inversion routine has placed all of the slip near the surface, resulting in a lower moment limit of $0.72 \times 10^{20} \mathrm{Nm}$, while in the upper panel most of the slip is placed deep on the fault plane to try to "hide" it from the surface data, resulting in an upper moment limit of $1.5 \times 10^{20} \mathrm{Nm}$. 
slip models. Geodetic data are less able to constrain deep fault slip as opposed to shallow slip, simply because of the larger distances involved, and so the amount of slip which can be hidden from the surface data increases as the fault deepens. Our 15-km depth limit was chosen based on seismological (Hauksson et al., 1993) as well as historical evidence for the southern California region.

\section{Results for a Large Geodetic Network}

Figure 5 presents the large network configuration used in the second part of this study. The data, in this case, consist of the three-dimensional co-seismic displacements for 24 GPS sites distributed throughout the nearfield region and tabulated in Hudnut et al. (1994), as well as the co-seismic line-length changes between the stations of the USGS Geodolite trilateration network as reported by Murray et al. (1993) and corrected for secular deformation as discussed by Hudnut et al. (1994). We have not included any of the far-field deformation data contained in Hudnut et al., to avoid possible difficulties from layering effects as briefly discussed in Bock et al. (1993). From this combined data set we have removed 13 vertical displacement measurements which have estimated errors of more than $600 \mathrm{~mm}$-these defor-

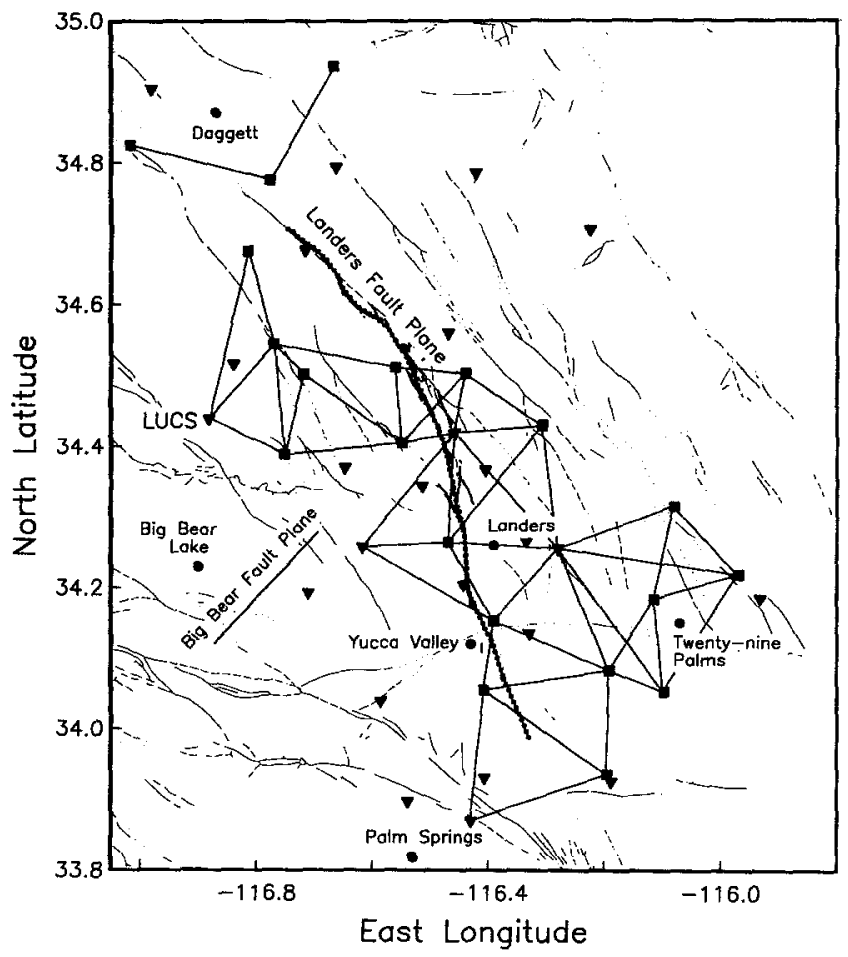

Figure 5. Geodetic stations used in what we call the "large network." Stations shown as squares and connected by solid lines are part of the USGS trilateration network, while stations shown as triangles were measured using GPS. Five stations were both part of the trilateration network and monitored with GPS. mations were very poorly constrained owing to the nature of the pre-earthquake GPS surveys, which were not conducted with precise geodetic applications in mind. With these removed we are left with 106 data.

In addition to these 13 vertical displacement data, we found another five data which we regard as questionable. All five data are related to the station LUCS in the western part of the network (Fig. 5). In the course of trying to model the data, we found we had great difficulty in this part of the network. This problem was also noted by Murray et al. (1993) who resolved the problem by artificially increasing the uncertainties for several measurements in the area. Their tentative conclusion was that unmodeled slip on the Upper Johnson Valley fault was responsible for this misfit. We believe, however, that the problem lies with the station LUCS. The station $\log$ sheets from this site for the postearthquake GPS occupations contain notes suggesting that the benchmark had been recently disturbed (J. Scott, personal comm.). At the time, there was no way to know when this had occurred, but we now believe that the benchmark (which is set in a concrete pillar projecting about $40 \mathrm{~cm}$ aboveground) was hit by a vehicle sometime between the preearthquake and postearthquake surveys. Our evidence comes from the model residuals for these five data, which suggest that the co-seismic displacement of station LUCS in the north-south direction is much too small. To de-

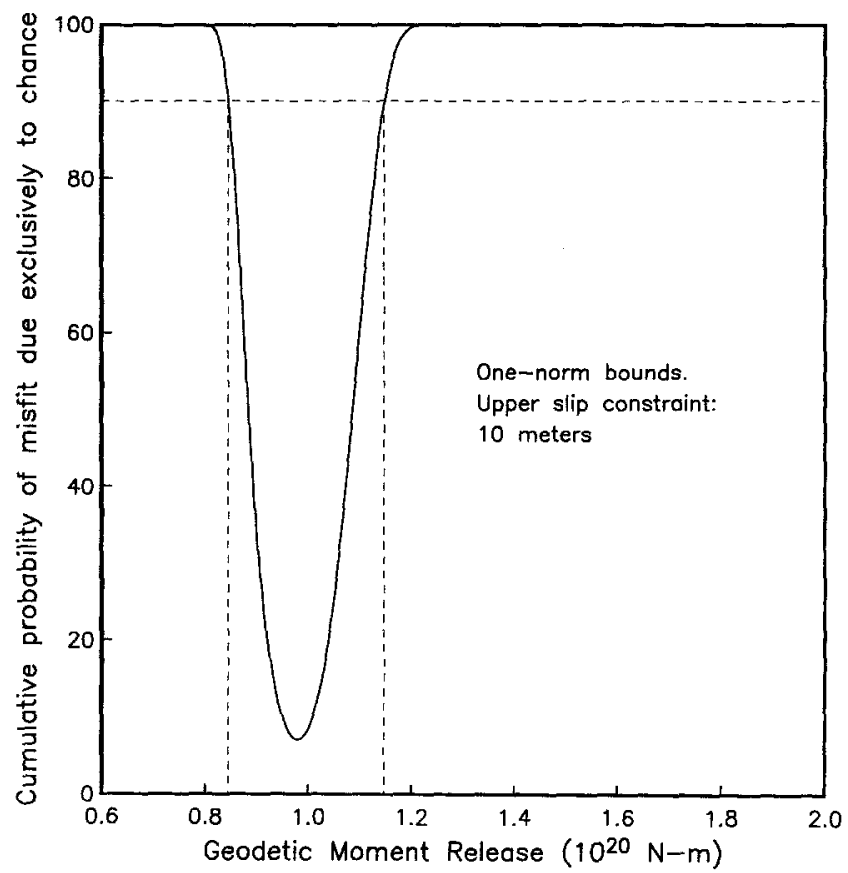

Figure 6. Slip-model probability plotted as a function of the total geodetic moment release for the large network. Only results for an upper slip constraint of $10 \mathrm{~m}$ are plotted. The scales here are the same as Figure 2. The bound on acceptable moment release is now considerably tighter than for the small network, resulting in a $90 \%$ confidence interval of 0.84 to $1.15 \times 10^{20} \mathrm{Nm}$ (M 7.25 to 7.34 ). 
termine this, we first removed the five data and calculated a best-fit slip model. This model was then used to predict what these five data should have been in order to agree with the other 101 data. By comparing the predicted data with the actual observations, which consisted of the NS and EW displacements from GPS and the changes in line length for three Geodolite lines, we are able to conclude that the benchmark was moved (nontectonically) to the south by about $5 \mathrm{~cm}$ and to the west by about $1 \mathrm{~cm}$. These values are in good agreement with the sketches of apparent disturbance in the GPS log sheets. In the end we feel there is sufficient evidence to remove these data from the inversion problem. With these data removed, there is no need to invoke slip on other faults. This is not to say that no slip occurred on other faultsthere is, for example, good evidence for at least some surface slip on the Lenwood fault (Padgett and Rockwell, 1993; Massonnet et al., 1994)-only that this collection of geodetic data does not require slip on any other faults to adequately fit the data.

Since we are now working with a much more extensive network of stations, the complication of the Big Bear earthquake must be dealt with in a different manner. Instead of ignoring this event, as was done before, we have chosen to model it and remove the model-pre-

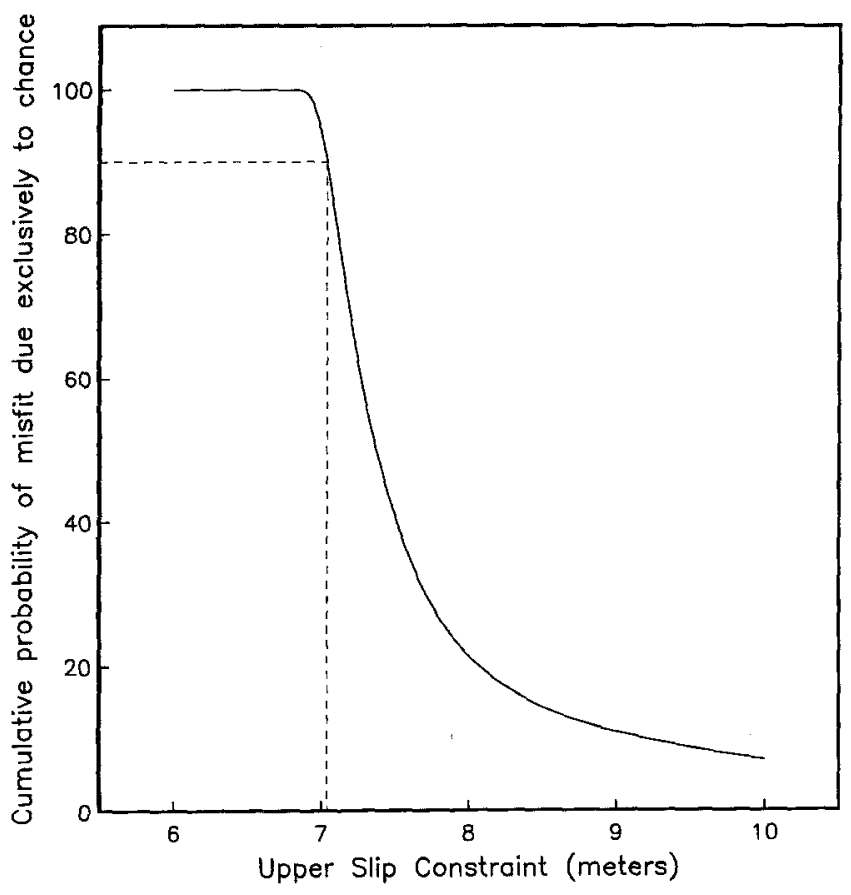

Figure 7. Lower bound on the maximum amount of fault slip required by the large network. Smaller upper-slip constraints on the fault model lead to poorer fits to the measured surface deformation data. As this limit is lowered past $7 \mathrm{~m}$, the best-fitting slip model results in a model misfit above the $90 \%$ level. From this we conclude (at a $90 \%$ level of confidence) that the $7 \mathrm{~m}$ upper-slip constraint is a bound and that at least one fault patch must have slipped more than this amount. dicted displacements from the data before inverting for the moment constraints on the Landers event. The model we use is based on the aftershock distribution shown in Hauksson et al. (1993). We chose a vertical fault plane which passes through the hypocenter of the event and extends from $1 \mathrm{~km}$ below the surface to a depth of 15 $\mathrm{km}$, with an along-strike dimension of $25 \mathrm{~km}$ and an azimuth of N43.4E. On this fault plane we place 0.35 $\mathrm{m}$ of left-lateral slip, which results in a moment release of $0.037 \times 10^{20} \mathrm{Nm}(\mathbf{M} 6.3)$. This makes our model Big Bear earthquake slightly larger than the value determined from seismic evidence by Hauksson et al. (1993). In fact, if we simultaneously invert the 101 geodetic data for the best-fitting slip model on both the Landers and the Big Bear fault planes, we would be led to an even larger moment release for the Big Bear event. That is, the data set used here would suggest that the amount of slip on the Big Bear fault were larger than the seismological results give. This is at least partly because we have used almost no data from the vicinity of the Big Bear event and so the amount of slip on this fault is poorly constrained.

With the data set thus cropped and the effects of the Big Bear earthquake removed, we can now proceed to the moment constraints for the Landers event. This result is presented in Figure 6 for the case of a 10-m slip constraint on all patches on the Landers fault-this can be directly compared to the results in Figure 2 . The bounds on the acceptable moment release for the event are now considerably tighter than before, with the $90 \%$ confidence limits being 0.84 to $1.15 \times 10^{20} \mathrm{Nm}$ (M 7.25 to 7.34). In calculating the results in Figure 6, we have continued to use the one-norm as our measure of model misfit. We chose to continue with the one-norm for two reasons. First, so that the results in Figure 6 would be directly comparable to Figure 2, and second, because of our suspicion that there may still be undetected measurement errors or even monumenting problems (as with station LUCS) left in the data. By using the one-norm measure of misfit, we are stating our belief that the measurement errors from both the GPS and the trilateration data are best described by a double-exponential distribution, as opposed to the standard assumption of a Gaussian distribution. The double-exponential distribution is more "heavy-tailed" than the Gaussian, and in our opinion more representative of the errors expected in these real-world measurements.

Figure 7 presents our bounds on the minimum value of the maximum amount of slip on the Landers fault. With $90 \%$ confidence we can state that at least one patch of the Landers fault must have slipped at least $7 \mathrm{~m}$ during the earthquake. As with the results in Figure 6, the steepness of this curve means that the level of confidence we choose has only a small affect on this bound. Again, this can be compared to Figure 2, where we found a $90 \%$ bound of $4.9 \mathrm{~m}$. The largest amount of measured surface slip was about $6 \mathrm{~m}$ at Galway Lake Road (Sieh et al., 1993). It is 
not surprising that our slip limit is larger than the largest measured surface offset; in fact, it is quite reassuring that it is slightly larger. Because of the unconsolidated nature of the surface layers along the Landers surface rupture, we should expect the measured offsets to be lower limits on the actual amount of surface slip. In addition, it is quite reasonable to believe that the amount of fault slip at depth will be different from that at the surface.

\section{Conclusions}

The technique we have presented for bounding the geodetic moment of an earthquake is (we hope) a valuable addition to the available seismic techniques. Geodetic measurements have the unique ability to monitor the entire moment release, extending to the longest periods, and are thus able to "see" the entire spectrum of co-seismic deformations. From a limited and preliminary GPS data set, we have shown that the moment release of the Landers earthquake was between 0.72 and $1.5 \times 10^{20} \mathrm{Nm}$ (M 7.2 to 7.4 ) with $90 \%$ confidence. This bound demonstrates the ability of this technique to produce results soon after a large earthquake. With time, as additional data are collected and more exacting analyses are performed, this technique can place tighter bounds on the moment release, as demonstrated here with the larger data set. This data constrain the moment of the Landers earthquake to be within 0.84 and $1.15 \times 10^{20}$ $\mathrm{Nm}$ (M 7.25 to 7.34 ) with $90 \%$ confidence.

\section{Acknowledgments}

The authors would like to thank P. Stark and R. Parker for sharing their computer code to simulate linear programming with one-norm misfits using BVLS. This work was supported by the Southern California Earthquake Center and the National Science Foundation.

\section{References}

Bock, Y., D. C. Agnew, P. Fang, J. F. Genrich, B. H. Hager, T. A. Herring, K. W. Hudnut, R. W. King, S. Larsen, J. B. Minster, K. Stark, S. Wdowinski, and F. K. Wyatt (1993). Detection of coseismic deformation from the Landers earthquake sequence using continuous geodetic measurements, Nature 361, 337-340.

Du, Y., A. Aydin, and P. Segall (1992). Comparison of various inversion techniques as applied to the determination of a geophysical deformation model for the 1983 Borah Peak earthquake, Bull. Seism. Soc. Am. 82, 1840-1866.

Erickson, L. L. (1986). A three-dimensional dislocation program with applications to faulting in the earth, M.S. Thesis, Stanford University, Stanford, California.

Harris, R. A. and P. Segall (1987). Detection of a locked zone at depth on the Parkfield, California, segment of the San Andreas Fault, J. Geophys. Res. 92, 7945-7962.

Hauksson, E., L. Jones, K. Hutton, and D. Eberhart-Phillips (1993). The 1992 Landers earthquake sequence: seismological observations, J. Geophys. Res. 98, 19835-19858.

Hudnut, K. W., Y. Bock, M. Cline, P. Fang, Y. Feng, J. Freymueller, X. Ge, W. K. Gross, D. Jackson, M. Kim, N. E. King, J. Langbein, S. C. Larsen, M. Lisowski, Z.-K. Shen, J. Svarc, and J. Zhang (1994). Coseismic displacements of the 1992 Landers earthquake sequence, Bull. Seism. Soc. Am. 84, no. 3, 625645 .

Langbein, J. (1981). An interpretation of episodic slip on the Calaveras fault near Holister, California, J. Geophys. Res. 86, 4941-4948.

Lawson, C. and R. Hanson (1974). Solving Least Squares Problems, Prentice-Hall, Englewood Cliffs, New Jersey.

Massonnet, D., K. L. Feigl, M. Rossi, and F. Adragna (1994). Radar interferometric mapping of deformation in the year after the Landers earthquake, Nature (submitted for publication).

Matthews, M. V. (1991). On the estimation of fault slip in space and time, Ph.D. Thesis, Stanford University, Stanford, California.

Murray, M. H., J. C. Savage, M. Lisowski, and W. K. Gross (1993). Coseismic displacements: 1992 Landers, California, earthquake, Geophys. Res. Lett. 20, 623-626.

Okada, Y. (1985). Surface deformation due to shear and tensile faults in a half-space, Bull. Seism. Soc. Am. 75, 1135-1154.

Padgett, D. and T. Rockwell (1993). Timing of past earthquakes and triggered slip events on the Lenwood fault at Soggy Lake Playa relative to 1992 Landers triggered slip, $\operatorname{EOS~74,68.~}$

Parker, R. and M. McNutt (1980). Statistics for the one-norm misfit measure, J. Geophys. Res. 85, 4429-4430.

Parker, R. L. (1994). Geophysical Inverse Theory, Princeton University Press, Princeton, New Jersey.

Press, W., B. Flannery, S. Teukolsky, and W. Vetterling (1987). Numerical Recipes, Cambridge University Press, Cambridge, United Kingdom.

Segall, P. and R. Harris (1987). Earthquake deformation cycle on the San Andreas Fault near Parkfield, California, J. Geophys. Res. 92, 10511-10525.

Shimada, S. and Y. Bock (1992). Crustal deformation measurements in central Japan determined by a global positioning system fixedpoint network, J. Geophys. Res. 97, 12437-12455.

Sieh, K., L. Jones, E. Hauksson, K. Hudnut, D. Eberhart-Phillips, T. Heaton, S. Hough, K. Hutton, H. Kanamori, A. Lilje, S. Lindvall, S. F. McGill, J. Mori, C. Rubin, J. A. Spotila, J. Stock, H. K. Thio, J. Treiman, B. Wernicke, and J. Zachariasen (1993). Near-field investigations of the Landers earthquake sequence, April to July 1992, Science 260, 171-176.

Stark, P. B. and R. L. Parker (1993). Bounded-Variable least-squares: an algorithm and applications, Technical Report No. 394, Department of Statistics, University of California Berkeley, Berkeley, California.

Vasco, D. (1985). Extremal inversion of vertical displacements, Long Valley Caldera, California 1982/1983, J. Geophys. Res. 57, 178183.

Wagner, H. (1959). Linear programming techniques for regression analysis, J. Am. Stat. Assoc. 54, 206-212.

Ward, S. N. and S. E. Barrientos (1986). An inversion for slip distribution and fault shape from geodetic observations of the 1983, Borah Peak, Idaho, earthquake, J. Geophys. Res. 91, 4909-4919.

Institute of Geophysics and Planetary Physics

Scripps Institution of Oceanography

University of California San Diego

La Jolla, California 92093-0225

(H.O.J., D.C.A.)

United States Geological Survey

Pasadena, California 91106

(K.H.)

Manuscript received 29 July 1993. 